\title{
LA CONSTRUCCIÓN DE CONTENIDOS INFORMATIVOS A PARTIR DE LA ÉTICA DEL PERIODISTA GUAYAQUILEÑO 3.0
}

\author{
THE CONSTRUCTION OF INFORMATIVE CONTENT BASED ON \\ THE ETHICS OF THE GUAYAQUIL JOURNALIST 3.0
}

\section{KARLA LÓPEZ CEDEÑO', MARÍA FERNANDA COTERA GRACIA ${ }^{2}$}

1 Universidad Laica Vicente Rocafuerte. kclopezc@ulvr.edu.ec

2 Universidad Laica Vicente Rocafuerte. mafercotera27@gmail.com
La presente investigación se orienta hacia el análisis de la ética periodística en los portales web de los diarios El Universo y El Comercio y su incidencia en la construcción de normativas deontológicas del periodista guayaquileño 3.0, por ello se abordó desde un enfoque mixto, que acopla técnicas cuantitativas y cualitativas, en un ambiente de campo, en el cual los objetos de estudio dieron su opinión y perspectiva respecto a la ética, el correcto uso de las normas deontológicas y la influencia de la información emitida por dichos medios. Se aplicó una encuesta a una muestra intencional de siete periodistas en el diario El Comercio y 10 periodistas de diario El Universo. Además se entrevistó a dos periodistas de los portales digitales: La República.ec y la Historia.ec, que antes laboraron en medios tradicionales. Tras aplicar las técnicas de recolección de datos como la entrevista y las encuestas se determinó que el $85 \%$ de la muestra considera importante que toda información emitida por los periodistas debe estar ligada a la ética periodística, que esta no debe perderse 0 ser sometida por los beneficios personales del comunicador o el medio de comunicación por el que se trasmiten las noticias. Asimismo, se debe educar a los nuevos periodistas para que sean íntegros en su carrera profesional ante la construcción de normativas deontológicas del periodista guayaquileño 3.0. Por su parte las entrevistas a profesionales definieron que, la ética periodística es parte del ejercicio profesional y no hay que desvincularse de ella. Además evidencian que en el país no existe una organización que se encargue de formar la ética periodística.

PALABRAS CLAVE: medios digitales, noticias, normativas deontológicas, ética periodística, veracidad informativa.
The present investigation is oriented towards the analysis of journalistic ethics in the web portals of the El Universo and El Comercio newspapers and its impact on the construction of deontological regulations of the journalist from Guayaquil 3.0, so it was approached from a mixed approach, which combines techniques quantitative and qualitative, in a field environment, in which the objects of study gave their opinion and perspective regarding ethics, the correct use of ethical standards and the influence of the information issued by these means. A survey was applied to an intentional sample of seven journalists in the newspaper El Comercio and 10 journalists of the newspaper El Universo. In addition, two journalists from the digital portals were interviewed: La República.ec and Historia.ec, who previously worked in traditional media. After applying data collection techniques such as interviews and surveys, it was determined that $85 \%$ of the sample considers it important that all information issued by journalists should be linked to journalistic ethics, which should not be lost or submitted by personal benefits of the communicator or the means of communication by which the news is transmitted. Likewise, new journalists should be educated to be integral in their professional career before the construction of deontological regulations of the journalist from Guayaquil 3.0. On the other hand, interviews with professionals defined that journalistic ethics is part of professional practice and should not be separated from it. They also show that there is no organization in the country that is responsible for forming journalistic ethics.

KEYWORDS: digital media, news, deontological regulations, journalistic ethics, informational veracity. 
INTRODUCCIÓN

El tema de la ética periodística es de gran importancia en el ejercicio de la carrera de comunicación social y en el contexto de la sociedad digital o de la información cuya expansión es acelerada. La noticia puede llegar a puntos de ubicación geográfica que antes parecían inaccesibles. Las nuevas plataformas y dispositivos tecnológicos le dan mayor peso a la inmediatez. Las investigaciones que abordan este tema, contribuyen a que los mecanismos y acciones enfocadas al periodismo digital, evolucionen en el tiempo. En este sentido, el análisis de los contenidos informativos de la versión digital de diario El Universo y El Comercio ante la gestión y construcción de normativas deontológicas del periodista guayaquileño 3.0, constituye un grandioso aporte desde la perspectiva de la praxis periodística en la actualidad, con la cual se busca profundizar en diversas experiencias de países que han tratado el tema de la ética periodística.

\section{REVISIÓN TEÓRICA ACERCAMIENTO TEÓRICO DEL PERIODISMO}

El periodismo es una institución que involucra procesos amplios de construcción de significados, de vinculación con los discursos, y cómo se vincula con los hechos que ocurren en la sociedad, jamás es estático, por la necesidad de interpretación de la información, y crea estructuras discursivas coherentes respecto a fenómenos o determinados hechos.

Ruiz, (2014) señala que la identidad del periodismo como profesión se fundamenta en el supuesto "lo creo cuando lo veo" (p. 25), agrega además que se trata de una profesión cuya brecha es muy amplia considerando su gran importancia en la sociedad, así como todo lo que involucra en su estructura, función y desarrollo de competencias en el campo. Pero más allá de eso, es todo un desafío para quien ejerce la carrera, porque en el desarrollo del periodismo mucho se cuestiona lo que se publica, los hechos, el ámbito, contenido, la política editorial del medio, en fin, es allí donde se pone de manifiesto la identidad del periodista.

Bajo estas ideas, el autor destaca sobre el ejercicio del periodismo que "relatar acontecimientos, informar hechos novedosas y al mismo tiempo discernir la verdad fáctica, son los principales elementos que definen el periodismo como una práctica cultural" (p. 26)

La práctica profesional del periodismo implica un ejercicio muy cauteloso que, en muchas circunstancias, maneja datos de fuen- tes oficiales y públicas, así como datos, cuyas fuentes no pueden ser reveladas, de manera que la documentación y/o fundamentación es indispensable para constituir la noticia.

En un estudio desarrollado por la Fundación Konrad Adenauer Stiftung, año 2016, sobre los perfiles profesionales de los periodistas en las democracias de América Latina, señalan en cuanto a la orientación ética de los periodistas en Ecuador, que estos demuestran un gran compromiso ético profesional, sin embargo, el estudio destaca que:

Más de la mitad de los periodistas admiten que su trabajo está influenciado por factores internos como, en un primer grupo, las políticas editoriales, los jefes directos y editores del medio de comunicación, los altos directivos del medio de comunicación y los propietarios del medio de comunicación, consecuencia de la estructura jerárquica y vertical dentro de los medios de comunicación; las redes sociales, fruto de la incorporación progresiva de las herramientas digitales al trabajo profesional periodístico; y los plazos y las restricciones de tiempo, prueba de que los procesos están definidos cada vez en mayor medida por la inmediatez y la primicia (Amado, Oller-Alonso, \& Baumgratz, 2016)

Más adelante, el estudio señala respecto a la percepción que tienen los periodistas ecuatorianos sobre sus roles profesionales es que, "en Ecuador se encuentran los periodistas inclinados a difundir la posición del gobierno", pero además, el estudio enfatiza los perfiles profesionales de los periodistas de acuerdo a tres dimensiones: intervencionismo, distancia del poder y orientación del mercado (Amado, Oller-Alonso, \& Baumgratz, 2016, pág. 25).

\section{COMUNICACIÓN (ESTRUCTURA Y FUNCIÓN)}

La comunicación surge hoy en día como una razón comunicacional que, de acuerdo con Martín-Barbero (2003) "sus dispositivos -la fragmentación que disloca y descentra, el flujo que globaliza y comprime, la conexión que desmaterializa e hibrida- agencian el devenir mercado de la sociedad" (pág. xiii). Entonces para el autor la comunicación se convierte en el más eficaz motor del desenganche e inserción de las culturas -étnicas, nacionales, o locales- en el espacio/ tiempo del mercado y las tecnologías globales.

En ese mismo sentido, se necesita pensar el lugar estratégico que ha pasado a ocupar la comunicación en la configuración de los nuevos modelos de sociedad, y su paradójica vinculación, tanto al relanzamiento de la 
modernización-vía satélite, informática, video procesadores- como a la desconcertada experiencia de la tarda modernidad.

De acuerdo con los autores Cerbino, Chiriboga, y Tutivén (2001), desde finales de los años 90 ha existido una emergencia de fenómenos sociales vinculados a los procesos de comunicación mass-mediáticos y de telemática, que están cambiando la modalidad en que se forman los grupos y el reconocimiento de las lógicas de producción de la identidad. Explican Cerbino, Chiriboga, \& Tutivén (2001), que "las formas de vida, adquieren otras sensibilidades frente al mundo político, económico y cultural e incluso, las interacciones simbólicas e imaginarias de la socialización han sufrido un cambio que incide tanto en los medios como en los procesos de comunicación social” (pág. 20)

La comunicación para estos autores:

Ya no es cuestión de medios sino también de mediaciones, es decir, que lo que más importa a esta mirada comunicacional no es tanto saber qué pasa exclusivamente en la racionalidad de los productores -la industria cultural, medios masivos de comunicación social, etc., - sino lo que está pasando, además, en las racionalidades conscientes o espontáneas de los consumidores y ciudadanos. Lo que está configurado son las modulaciones del consumo desde lo local, o desde las micro escenas de las vidas cotidianas, o desde las modas que "posicionan" a los sujetos en una lógica de reconocimiento entre diferencias. Asistimos, así, a un efecto de recursividad en la dialéctica entre medios y mediaciones (Cerbino, Chiriboga, \& Tutivén, 2001, pág. 20).

En este ambiente, los actores sociales son agentes y pacientes de los productores informáticos de los medios de comunicación y éstos, a su vez, reconfiguran el campo del consumo de la información y de la comunicación social.

LA ÉTICA EN EL PERIODISMO

La ética se refiere a los principios o reglas de conducta y prácticas deseables que orientan el comportamiento del individuo, en este caso, el profesional del periodismo. Sus valores se orientan hacia el empirismo, según el cual, maneja la información con carácter veraz. De allí que, los principios básicos sobre los cuales debe trabajar el periodista son: verdad y precisión, independencia, equidad e imparcialidad, humanidad y responsabilidad. (Ethical Jouirnalism Network, 2018).

El manejo ético de la información hace referencia a la verdad con que se maneja la infor- mación, la misma constituye el punto central del trabajo del periodismo, es aquel en el cual se da un tratamiento veraz y objetivo, se respeta el derecho y la dignidad de las personas.

Y el impacto es la forma en la que se recibe la información por parte de la ciudadanía, su incidencia, en la forma de pensar y actuar, o la forma de responder ante la información que consume.

\section{CÓDIGOS DEONTOLÓGICOS}

Son aquellos códigos que implican el tratamiento ético de la información. En tal sentido, los principios fundamentales o códigos éticos para el ejercicio de cualquier profesión, y en especial, para el ejercicio periodístico son de suma importancia. Subsiguientemente se exponen los Códigos Deontológicos de los medios periodísticos en función de establecer una estructura ética como sustento de la presente investigación. Por tanto, los códigos deontológicos se pueden definir como el conjunto de reglas y normas que guían u orientan la conducta del periodista, le dan un carácter de honestidad y honorabilidad a quien ejerce esta labor. Estos por lo general, se componen de los deberes, objetivo de la profesión, su actuación hacia sus compañeros, algunas restricciones para casos específicos, en el manejo de fuentes. El código de ética del profesional de la comunicación es importante en el desarrollo de la noticia, toda vez que este se encuentre comprometido con la misma.

Bajo estas ideas, se refiere el Código de Ética del Periodista de la República de Ecuador, cuya Federación ha orientado una Normativa para orientar la actividad profesional en la carrera periodística, la cual coincide con los dos documentos referidos anteriormente, en lo que rezan sus artículos: 25, 30, 32, respecto a la veracidad de la información, apropiarse de información ajena, y lo que se agrega es que cualquier información que se publique no debe ir detrimento de la dignidad de la persona, instituciones o grupos.

\section{TEORÍA HISTÓRICA DE LA EVOLUCIÓN DE LOS MEDIOS EN LA ERA DEL INTERNET}

Uno de los primeros medios que dieron el salto de los medios tradicionales a internet, fue el diario brasileño "Jornal do Brasil", en el mes de mayo del año 1995 en la ciudad de Rio de Janeiro, esta fue una de las primeras ediciones vía web de un diario Latinoamericano y la primera en el país carioca, vale destacar que dicho diario estuvo en un proceso de adaptación web por aproximadamente 8 meses. 
Esto sirvió como un empuje para los demás medios en los diferentes países ubicados en la parte sur del continente americano, pues en aquel año otros ejemplares dieron lugar a la transición de los portales web, donde obtuvieron como resultado, la ventaja de dar información en tiempo real sobre un acontecimiento de relevancia para todo un país a través de la plataforma web. Suceso impensable décadas atrás. Específicamente, hay 22 países que conforman la región iberoamericana, de acuerdo al autor Salaverria (2015)

En once de estos veintidós países Argentina, Bolivia, Chile, Costa Rica, Ecuador, El Salvador, México, Nicaragua, Panamá, Perú y Uruguay, dieron el primer salto a los medios digitales en una fecha incluida entre el 1 de enero y el 31 de diciembre de 1995. Solo España, Portugal y Brasil vieron los medios digitales aparecer antes: durante 1994, cuando un puñado de medios lanzaron versiones rudimentarias en línea que ni siquiera se publicaron inicialmente en el "World Wide Web ", pero en varias redes anteriores. (Salaverria, 2015, pág. 11)

En los ocho países restantes Colombia, Cuba, Guatemala, Honduras, Paraguay, Puerto Rico, República Dominicana y Venezuela, los primeros medios digitales datan de 1996. Por lo tanto, y a pesar de estas variaciones, los medios digitales iberoamericanos en su conjunto alcanzaron en 2015 su vigésimo aniversario, a continuación, se procede a mostrar una tabla de los primeros medios dieron el salto de los medios tradicionales a internet. (Salaverria, 2015, pág. 11)

Internet no es una simple red de ordenadores, es una red de redes, es decir, un conjunto de redes interconectadas, a escala mundial con la particularidad de que cada una de ellas es independiente y autónoma (Avila, 2007, p. 22). El mismo autor explica que el Internet fue creado a fines de los años 60, aproximadamente para el año 1983, por el Departamento de Defensa de los Estados Unidos, como una red experimental llamada ARPANET (Advanced Research Projects Agency Network, Red de la Agencia de Proyectos de Investigación Avanzada), a partir de ese momento evoluciona de manera muy rápida.

De la mano a estos avances tecnológicos, los medios de comunicación han evolucionado en todos los países de mundo, con distintas estrategias y multiplicando las posibilidades comunicativas y con fines no imaginados. Se logra transmitir más información en los mismos canales, permitiendo la especialización de contenidos llegando a millones de personas en el mundo.

Los medios cumplen diferentes roles básicos en nuestra sociedad. Un papel es el entretenimiento. Los medios de comunicación pueden actuar como trampolín para nuestra imaginación, una fuente de fantasía y una salida para el escapismo. En el siglo XIX, los lectores victorianos desilusionados por la dureza de la Revolución Industrial se vieron atraídos hacia mundos fantásticos de hadas y otros seres ficticios.

\section{EL PERIODISMO EN LA WEB}

En relación a este tema, Morales, Lluiz, \& Tous (2016), manifiestan a través de su informe que "Detrás de la evolución de los flujos de contenidos mediáticos hay una serie de cambios tecnológicos clave" (p. 73). Ahora bien, es para los años 90 cuando se inician las apariciones de los primeros contenidos mediáticos de los usuarios. Los mismos autores señalan que es para el año 2005 cuando se generaliza la producción mediática propia y se comienza la aparición de la fase de la web 2.0.

Guadarrama (2007), realiza una revisión de los contenidos mediáticos en ocho países, a fin de ahondar con mayor confianza en el tema. En primer lugar, el autor toma como una de las referencias a Estados Unidos, como país que ha insistido en erradicar la violencia en los medios de comunicación, generando grandes esfuerzos para impulsar la clasificación de los contenidos de los programas, tanto en la televisión abierta como la restringida. (p. 75). En el caso señalado, la televisión abierta se refiere a los medios que ofrecen información para todos por cualquier medio en el que se transmita y en cuanto a la televisión restringida, esta se refiere a la televisión paga o televisión digital, donde el usuario debe cancelar por acceder a la programación.

En este orden de ideas, según los autores Osuna \& Busón (2012) el gobierno norteamericano comienza a establecer poco a poco medidas para clasificar los contenidos mediáticos en distintas áreas, como largometrajes, videojuegos, software para computadoras, discos musicales, considerando entre otros aspectos, la edad apropiada en la que las personas pueden hacer uso de la información.

Puntualizando un poco más acerca de los contenidos mediáticos en la web, se puede entender que, a pesar de la inmensa cantidad 
de información publicada a través de la red, se hace necesario que tal información sea confiable y que genere mayor credibilidad para el lector y el aprendiz.

En este sentido, los países han desarrollado normas y leyes que contribuyen al control estricto de los contenidos que se publican en ciertos medios, en especial los tradicionales contribuyendo esto a minimizar la divulgación de mensajes que atenten a la moral y a las buenas costumbres, así como la falsificación de información. Y con respecto a la web, Según Peman (2016) existen algunas características de importancia de la información que se publica en la web.

\section{ANÁLISIS DE RESULTADOS}

Luego de aplicar las encuestas a la muestra escogida de ecuatorianos, se obtuvieron los siguientes resultados:

- EL $100 \%$ de los encuestados permite evidenciar que la ética periodística es fundamental en el momento de ejercer la profesión; afirman que su importancia se basa al momento de realizar una correcta redacción de los hechos, sin alterar las noticias, ni sus fuentes y coinciden que la ética se encuentra asociado a valores humanos.

- El $52 \%$ de los periodistas encuestados manifestaron que algunos medios digitales no gozan de una buena imagen, ni credibilidad en su información publicada, mientras que el $47 \%$ de ellos opina que los medios digitales del país sí gozan de buena imagen y credibilidad ya que se han ganado ese espacio gracias a la trayectoria y su compromiso con la sociedad.

- Al analizar los factores principales que ponen en riesgo la ética periodística en el país, el $58 \%$ de los encuestados indicaron que se debe al limitado acceso de fuentes informativas, mientras que el $76 \%$ dijo que son los intereses del medio y la autocensura profesional, la influencia de la línea editorial del medio también influye como factor dejando como resultado el $64 \%$, el $35 \%$ de los encuestados indicaron que el factor es la restricción a la libertad de expresión y el $88 \%$ los intereses económicos o personales del periodista.

- El $100 \%$ de los periodistas encuestados del diario El Comercio Y El Universo consideran que el periodismo sí se fortalece a través de la ética, ya que es una base para todas las publicaciones de un medio, creando una reputación de credibilidad ante los lectores.

Entrevista a los periodistas de los portales web LaHistoria.ec y LaRepública.ec

A continuación se efectúa un análisis cualitativo con relación a las entrevistas dirigidas a dos periodistas de medios digitales, que expusieron su visión ante la ética periodística informativa en los procesos de comunicación digital de los diarios El Universo y El Comercio. Las personas entrevistadas fueron El periodista y fundador del portal web lahistoria.ec Marlon Puertas y el periodista y director de la plataforma digital larepública.ec Carlos Jijón representando experiencia en el tema.

- El periodista Marlon Puertas enfatiza que la ética debe ser generalizada, por ello indicó que es necesario crear espacios específicos para desarrollarla, para él es una lástima que en Ecuador no exista ninguna organización que fomente la ética periodística, también piensa que ningún gobierno en el pasado y mucho menos el actual está interesado en lo más mínimo en promover la ética al momento de emitir una información y no tiene la intención de fortalecer la libertad de expresión, además que los gobiernos no han tenido un plan o una organización destinada a la implementación de programas con la finalidad de la consolidación de la ética en el ejercicio del periodismo.

- En relación con la pregunta de que si él aportaría con una propuesta para el fortalecimiento de la ética periodística en la ciudad de Guayaquil, el entrevistado manifestó que en lo personal él no aportaría en esta medida, puesto que considera que la ética es algo que los periodistas deben tener ya con ellos y que eso no es algo que aprendan en un seminario o en un curso impuesto, ya que siente que así no es cómo funciona la ética, que si se tiene este valor se tiene y si no, no es algo que se pueda enseñar en un par de horas.

- Al pensar en el caso hipotético entre el periodista y el medio de comunicación en el que trabajan les sugiera u obligue 
a alterar la información de la noticia para que esta favorezca al medio, el entrevistado indicó que los periodistas deben rechazar cualquier intención de manipulación de la información, manteniendo una postura de que el interés particular y la mentira deben ser un indicador para que el periodista se retire de ese medio, si no le queda de otra, para no manchar su carrera.

- El periodista Carlos Jijón por su parte, nos deja la reflexión de que en el país si existen medios que manejan una ética impecable y profesional que tiene como base la ética periodística, lo cual es esencial para que el medio aumente su audiencia, él afirma que diario El Universo es un medio que tiene una trayectoria, al realizar un buen periodismo, el cual está fuertemente ligado a la ética periodística.

- En cuanto si él considera que el gobierno debe promover la ética informativa y libertad de expresión a través de la ley, afirma que el gobierno no tiene entre sus funciones promover la ética periodística de los medios de comunicación y que no está obligado de ninguna forma a implementar programas o leyes para que la ética sea ejercida. Bajo su criterio estima que es un trabajo neto de los periodistas y los medios de comunicación que la ética periodística sea llevado a cabo, que esta es la forma profesional por la cual deben manejarse y que de hacerlo correctamente, el medio seguirá con su trayectoria y conseguirá más audiencia que se base en información veraz y contrastada, sin ningún tipo de manipulación.

Él considera que si un periodista se ve irremediablemente obligado a manipular la información y emitirla de tal modo que le convenga al medio para el que trabaja este periodista debe ser fiel a su ética y al cumplimiento de su trabajo, que así es como podrá mantenerse íntegro en su labor. En cuanto al manejo de un ambiente periodístico considera que debe manejarse con ética por parte el medio y el periodista en cada noticia difundida hacia al lector.

\section{DISCUSIÓN Y CONCLUSIONES}

Se define que los contenidos informativos en las plataformas digitales estudiadas se apegan al manejo ético periodístico por sus años de experiencia y trayectoria, no obstante existen medios digitales que con el objetivo de obtener la primicia, dejan a un lado el contraste de la información y la veracidad, lo que en definitiva significa que no todos los medios digitales son éticos al momento de informar a la sociedad.

Las bases teóricas desarrolladas en el presente proyecto de investigación, evidencia que, la información emitida a través de los medios digitales, incide directamente, en la construcción del pensamiento de los ciudadanos de Guayaquil, por este motivo se elaboraron recomendaciones, para que publiquen noticias que se apeguen a la línea de ética periodística.

Los medios digitales no están sujetos a ningún tipo de ley u obligaciones, eso los lleva a ser más responsables a la hora de emitir criterios informativos, lo que significa que antes de subir a su plataforma cualquier tipo de información, esta primero debe pasar los filtros de control editorial, para revisar si la fuente que proporcionó la información es confiable.

Los profesionales encuestados en este trabajo de investigación, dan una fiel muestra de que la ética periodística es una base fundamental para ejercer de una manera profesional. Por su parte, los entrevistados consideraron que los periodistas que no estén ligados a este principio de su profesión, están propensos a mancillar su carrera al practicarla con poco profesionalismo.

El manejo de la información por parte de los medios digitales son una extensión de medios tradicionales y para continuar con este rol fundamental en la sociedad de informar, se requiere preservar los principios básicos como periodistas; de buscar la verdad, ser responsables y actuar con trasparencia ante la audiencia lectora. De esta manera se puede seguir fortaleciendo el valor de la ética, como principal valor fundamental en el periodista guayaquileño 3.0.

\section{REFERENCIAS BIBLIOGRÁFICAS}

Amado, A., Oller-Alonso, M., \& Baumgratz, M. (2016). El periodismo por los periodistas. Perfiles profesionales en las democracias de América Latina. Montevideo: Konrad Adenauer Stiftung. Retrieved from https://www.kas.de/c/document_library/get_file?uuid=ef1da7ad-6f7a835e-33bb-41a0798a6885\&groupId $=252038$ 
Avila, A. (2007, p. 22). Iniciacion a la Red de Internet. Retrieved from Iniciacion a la Red de Internet: https://books.google.com.ec/

Diaz-Campo, J., Chaparro-Dominguez, M. Á., \& Segado-Boj, F. (2016). Ética y Periodismo de datos en los códigos de los medios españoles. RIoja, España : Universidad Internacional de la Rioja.

Ethical Jouirnalism Network. (2018). ethicaljournalismnetwork.org. Retrieved Noviembre Jueves, 2018, from ethicaljournalismnetwork.org: https://ethicaljournalismnetwork.org/whowe-are/5-principles-of-journalism

International Center for Journalists. (2018). Ética Periodistica en la era digital . Washington DC : UNESCO.
López, G. (2015). Periodismo digital: Redes, audiencias y modelos de negocio. CALEIDOSCOPIO: Periodismo digital, 10, 175 -176.

Martín-Barbero, J. (2003). De los medios a las mediaciones: Comunicación, cultura y hegemonía (Quinta Edición ed.). Bogotá, Colombia: Convenio Andrés Bello.

Medina, A. (2017). Herramientas de la planifıcación estratégica para su aplicación en el ejercicio periodístico de los nuevos medios digitales en el país. Caso portal web del diario El Comercio, de Ecuador . Quito: Universidad Andina Simón Bolívar .

Miller, K. H. (2019, Septiembre 26). Ética periodistica. Conceptos y prácticas desde sus actores . Retrieved from www.dereojo.org: http://www.dereojo. org/omar/etica-conceptos.pdf 